\title{
VERIFICATION OF THE COMPUTED TOMOGRAPHY RESULTS OF ALUMINUM ALLOY WELDED JOINT
}

\author{
Maciej Malicki, Kamil Sobczak \\ Institute of Aviation, Warsaw, Poland \\ Maciej.malicki@ilot.edu.pl
}

\begin{abstract}
Computed tomography (CT) of aluminum welded joint specimen has been performed. On the tomographic cross sections some defects have been found. To verify them the metallography cross sections of welded has been done. It was found that selected defects are micro cracks.
\end{abstract}

Keywords: computed tomography (CT), welded joints, defects, micro cracks

\section{INTRODUCTION}

One way to evaluate the quality of welded joints is to perform nondestructive tests. One of the techniques available is the X-ray computed tomography (CT) [1]. CT tests allow researchers to detect external and internal defects of welded joints such as pores, voids and micro cracks, which can reduce the joints' strength. In this work, the CT of the welded joint made from the aluminum alloy 6061 was performed (Fig. 1). The weld was made in the same way as the one planned to be used in a tank to be later subjected to pressure tests. That is why the CT inspection is especially useful in the pre-qualification phase of a technology [2].

\section{EXPERIMENTAL TECHNIQUE}

For the CT scans, the Industrial X-ray computed tomography system v/tome x s240 was used (Fig. 2). The weld joined two parts of the pipe of the $100 \mathrm{~mm}$ diameter and $5 \mathrm{~mm}$ wall thickness. The two scans were made: for the whole welded joint and for the specimen cut from the weld being tested. The CT scans of a higher resolution were also done for the welded specimen to visualize the defects invisible in the CT image made at a lower resolution (Fig 3). The cut specimen contained an around $35 \mathrm{~mm}$ part of the weld with a heat affected zone 
(HAZ). Also, an EDX analysis was performed for one of the welded joint areas where the defects were found.

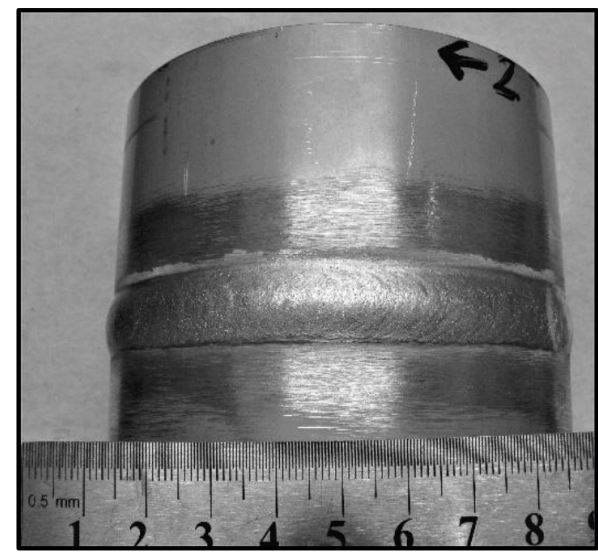

Fig. 1. Tested welded joint

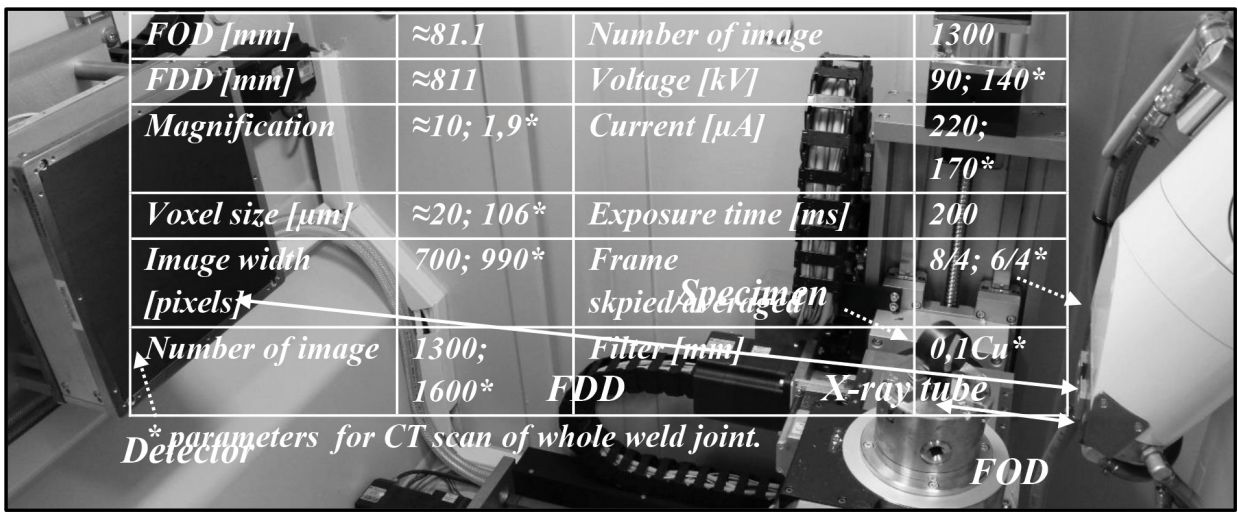

Figure 2. Measuring system

\section{RESULTS AND DISCUSSION}

The first CT scan of the whole welded joint (Fig. 3) revealed some defects in the form of linear areas $3 \mathrm{~mm}$ long with reduced as density compared with the parent material [3]. It was not possible to unequivocally state whether they were cracks or inclusions. To identify these defects the metallographic cross sections were made. It was found that in area containing defects on the CT cross sections, cracks were present (Fig. 4). These cracks ran along the one of the material phase. 


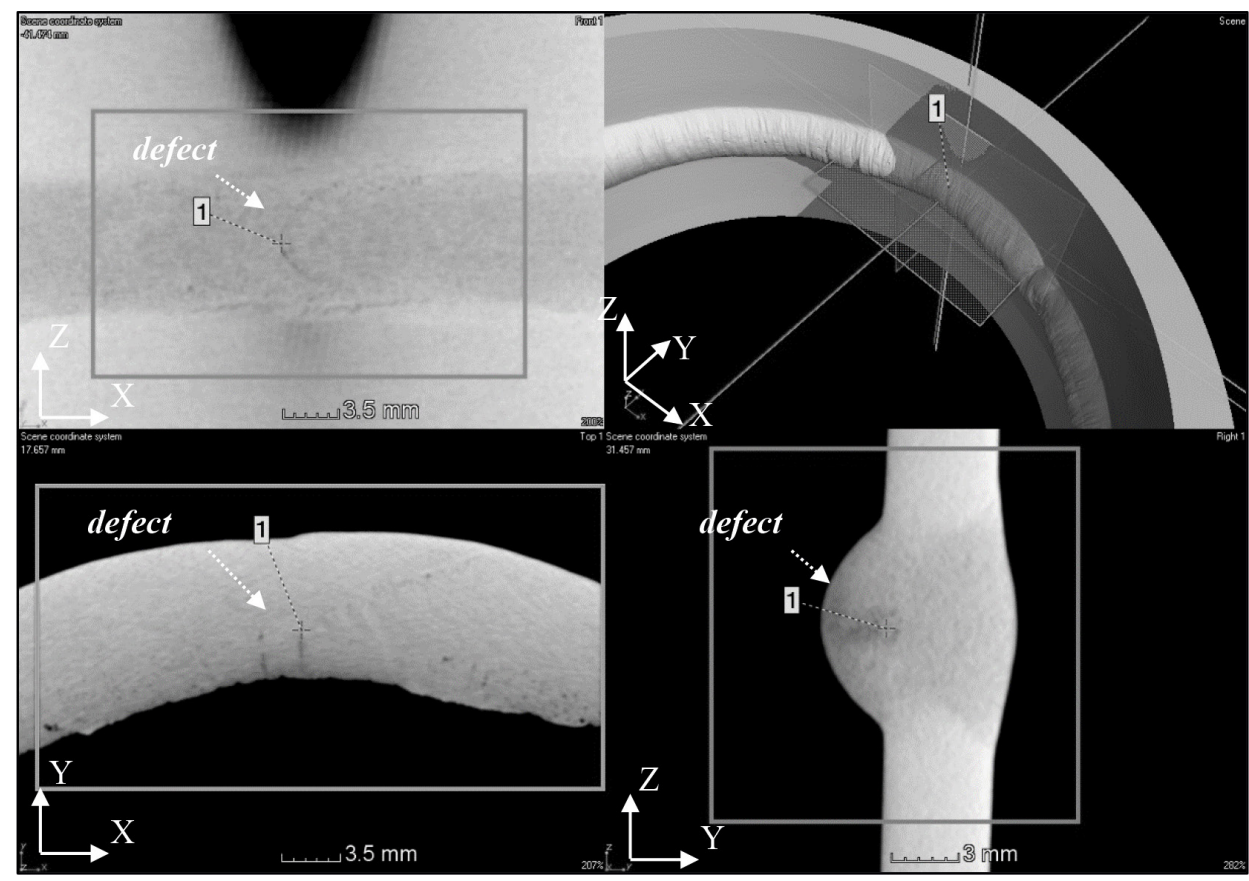

Fig. 3. CT Image of defects in tested welded joint
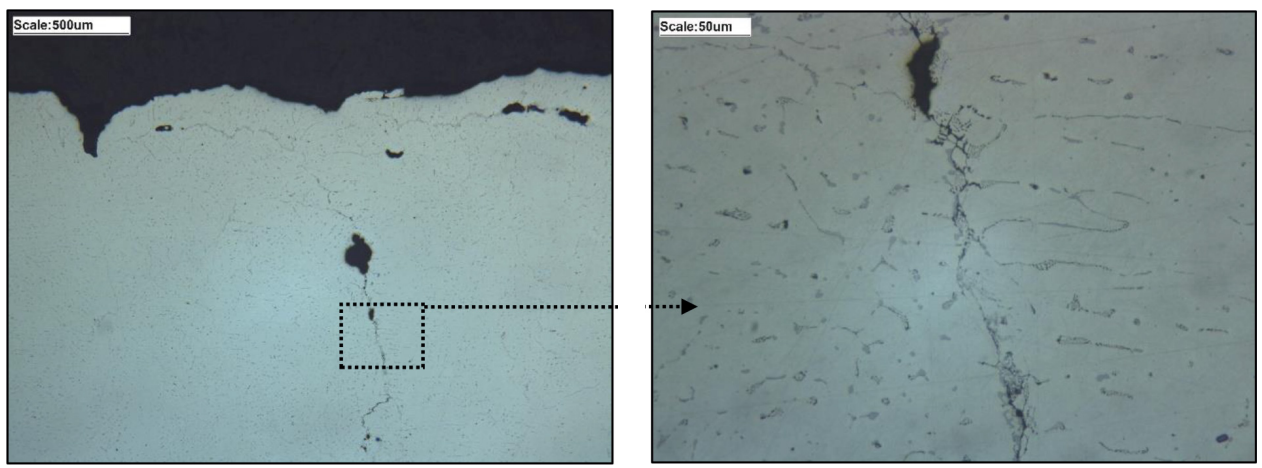

Fig. 4. Metallographic cross section of tested welded joint

The high resolution CT scan made for the welded specimen showed other defects too. These were less than $1 \mathrm{~mm}$ long and formed in the HAZ (Fig. 5). Also, the metallographic inspection made in the area corresponding to that defect, showed the present of cracks (Fig. 6, Fig 7). 


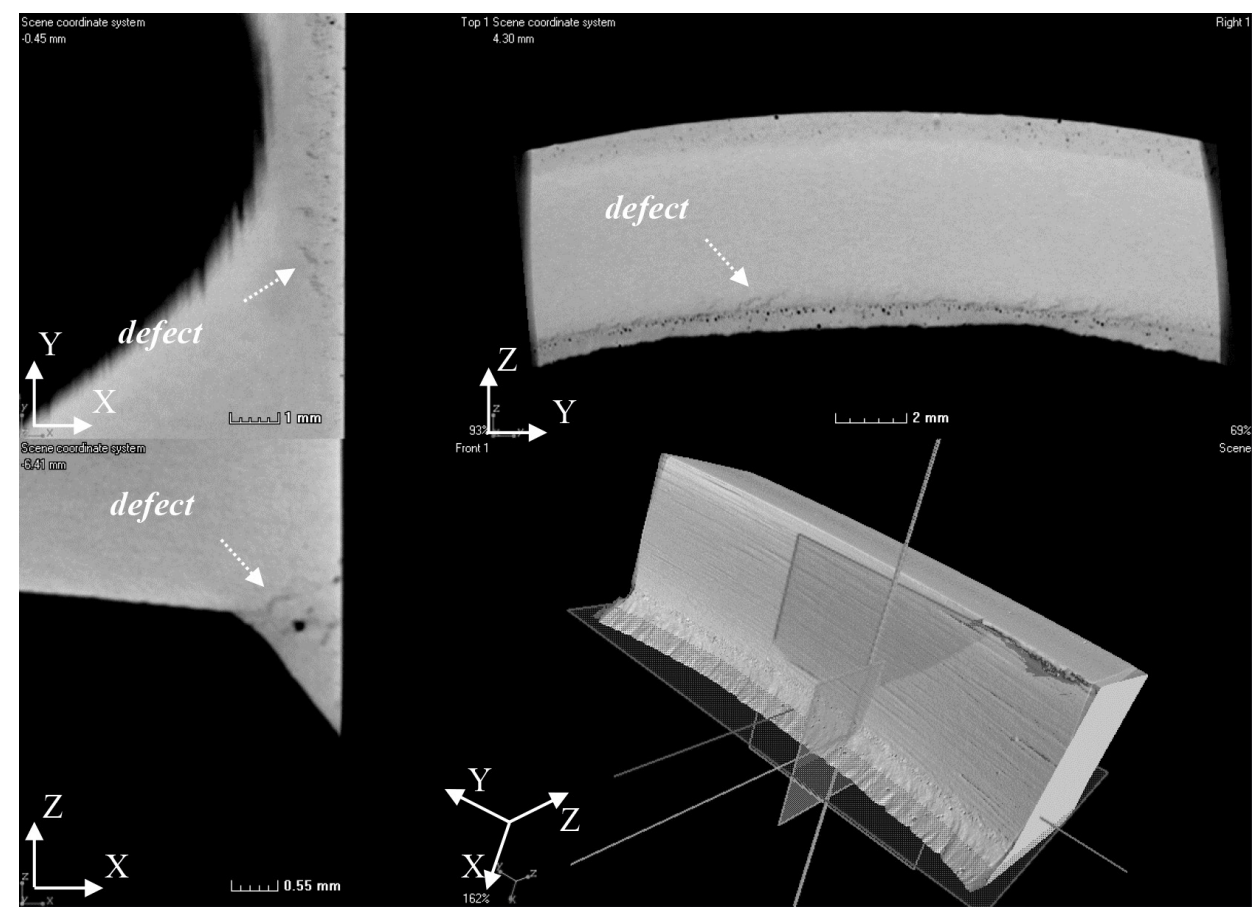

Fig. 5. CT image of welded joint specimen
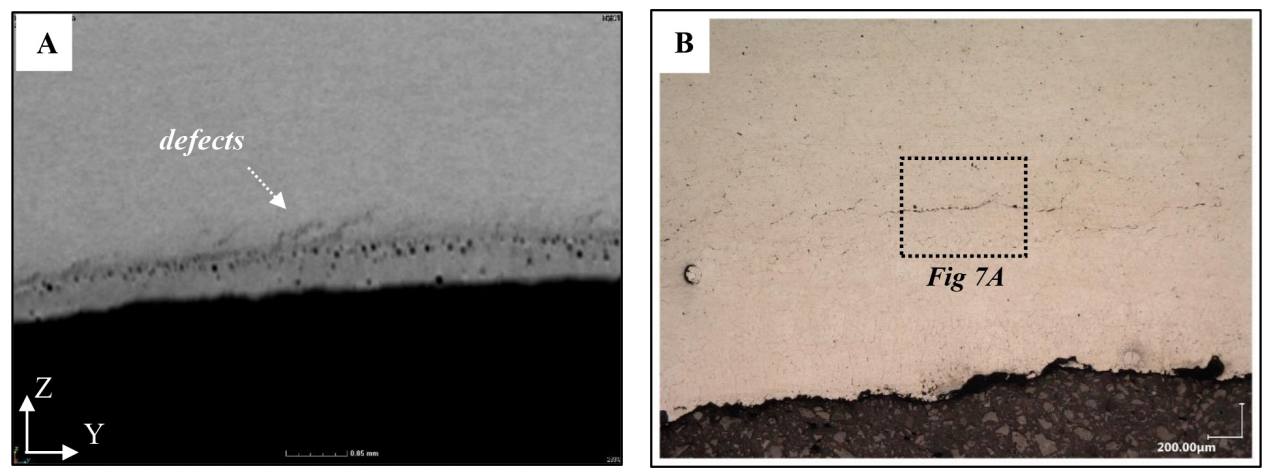

Fig.6. CT image of defects in welded joint specimen (A) Metallographic inspection made in area correspond to the defects $(B)$ 

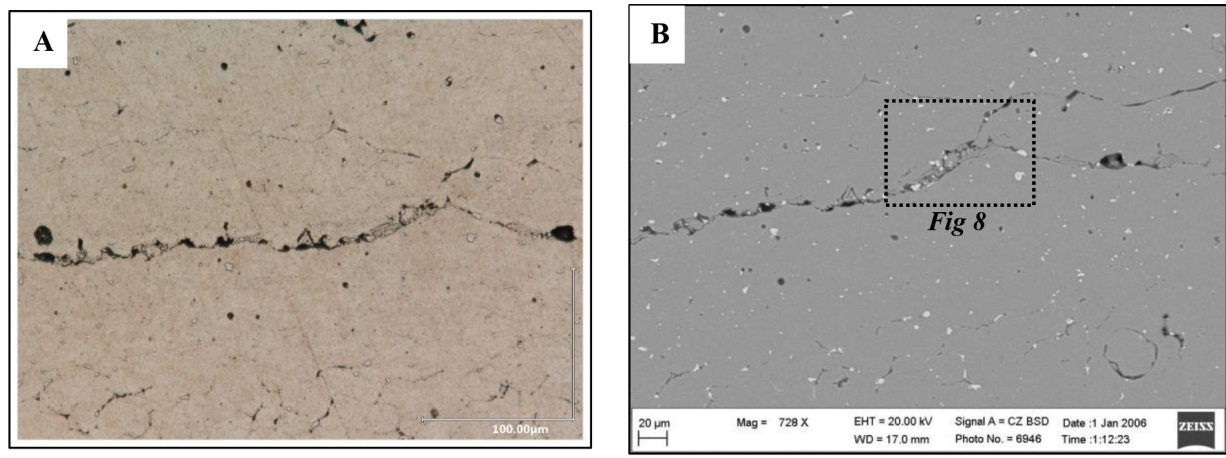

Fig. 7. Metallographic images of crack area. Optical microscope (A), scanning electron microscope using back scattering electron detector

An EDX analysis (Fig. 8) showed that in the crack area there was a material phase rich in iron, magnesium and silica (compared with the parent material). High temperature occurring during welding might well have caused the presence of these phases. The occurrence of precipitates combined with thermal stress could have been the source of the crack formation [4], [5].

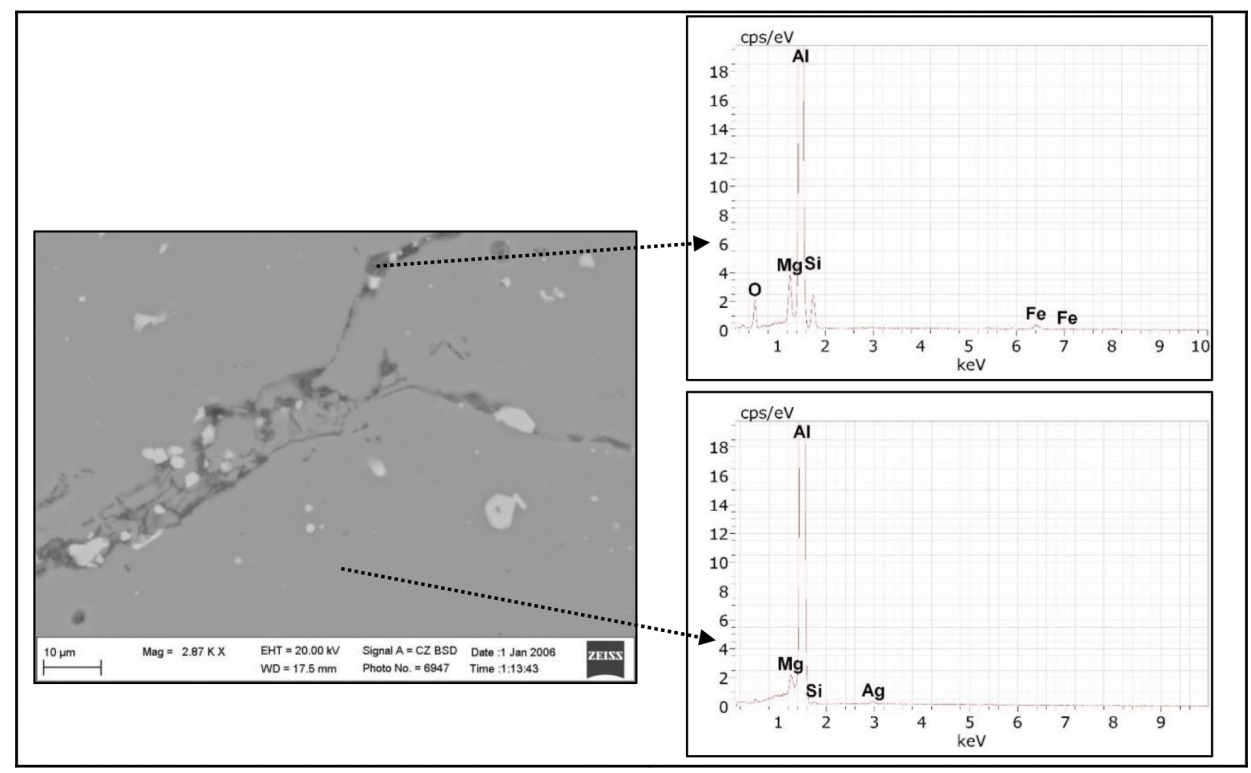

Fig.8. EDX analysis in crack area and in parent material 


\section{CONCLUSSIONS}

This study led to the following conclusions:

- material defects were detected in the welded joint using CT,

- defects detected were cracks propagating along the material phase or phases rich in iron, magnesium and silica (as compared with the parent material),

- those material phase or phases combined with thermal stress could have been the source of the crack formation.

\section{REFERENCES}

[1] R.F. Hamade , A.M.R. Baydoun, Nondestructive detection of defects in friction stir welded lap joints using computed tomography, Materials and Design 162 (2019) 10-23.

[2] ECSS Standard - ECSS-Q-ST-70-39C „Welding of metallic materials for light hardware", ECSS Executive Secretariat, ECSS Technical Authority, 1 Maj 2015

[3] Tomographic and metallographic tests protocol, CBMK/LM2/21935/06/ 1/2017, Institute of Aviation, CBMM Archives.

[4] N. Coniglio, C.E. Cross, I. Dörfel, W. Österle, Phase formation in 6060/4043 aluminum weld solidification, Materials Science and Engineering A 517 (2009) 321-327.

[5] W. Boonchouytan, J. Chatthong, S. Rawangwong, R. Burapa, Effect of Heat Treatment T6 on the Friction Stir Welded SSM 6061 Aluminum Alloys, Energy Procedia 56 ( 2014 ) $172-180$. 